\title{
Reviewers in this Issue
}

Prof. Shah Md. Zahurul Haque Asna

Department of Microbiology

Bangladesh University of Health Sciences, Dhaka

Prof. Mahmuda Begum Department of Pharmacology and Therapeutics

Enam Medical College, Savar, Dhaka

Prof. Md. Nur Hossain

Department of Forensic Medicine

Delta Medical College, Dhaka

Prof. Nasreen Chowdhury

Department of Biochemistry

Chattagram Maa-O-Shishu Hospital Medical College

Dr. Md. Khalilur Rahman

Associate Professor

Department of Radiology \& Imaging

Dhaka Medical College \& Hospital, Dhaka

Dr. Md. Rezwanur Rahman

Associate Professor

Department of Biochemistry

Delta Medical College, Dhaka

Dr. Irin Perveen

Associate Professor

Department of Gastroenterology

Enam Medical College \& Hospital, Savar, Dhaka

Dr. Rukhsana Parvin

Associate Professor

Department of Medicine

Enam Medical College \& Hospital, Savar, Dhaka 